PROCEEDINGS OF THE

AMERICAN MATHEMATICAL SOCIETY

Volume 139, Number 1, January 2011, Pages 21-30

S 0002-9939(2010)10660-3

Article electronically published on August 26, 2010

\title{
CENTRAL VALUES OF $L$-FUNCTIONS AND HALF-INTEGRAL WEIGHT FORMS
}

\author{
HUI XUE
}

(Communicated by Ken Ono)

\begin{abstract}
We prove a relation between the Fourier coefficients of certain Hilbert modular forms of half-integral weight and central values of the corresponding Rankin $L$-functions. The result generalizes the classical theorem by Waldspurger. The approach is geometric and generalizes that of Gross and Hatcher.
\end{abstract}

\section{INTRODUCTION}

Let $F$ be a totally real field of degree $d$. For the purpose of this paper the class number $h(F)$ of $F$ is always assumed to be odd. Let $f$ be a holomorphic Hilbert newform over $F$ of weight $2 \mathbf{k}=\left(2 k_{1}, 2 k_{2}, \cdots, 2 k_{d}\right)\left(k_{i} \geq 1\right)$ and level $N=\mathfrak{p}^{e}$ for a prime ideal $\mathfrak{p}$ of $F$ and $e \geq 1$. Assume further that if $d$ is odd, then so is $e$. Let $B$ be the quaternion algebra over $F$ such that:

(1) If $d$ is even, then $B$ is only ramified at all infinite places.

(2) If $d$ and $e$ are both odd, then $B$ is only ramified at infinite places and $\mathfrak{p}$. For $D \gg 0$ in $F$, let $\chi_{D}$ be the quadratic character of the idèle class group $\mathbb{A}_{F}^{\times} / F^{\times}$ associated to the quadratic extension $K_{D}=F(\sqrt{-D})$. Suppose that the conductor of $\chi_{D}$ is relatively prime to $\mathfrak{p}$ and that $K_{D}$ can be embedded in $B$, that is, $\chi_{D}(\mathfrak{p})=$ -1 . Write $L\left(s, f_{D}\right)=L(s, f) L\left(s, f \otimes \chi_{D}\right)$ for the (complete) $L$-function of $f$ over $K_{D}$. It is well known that the sign of the functional equation of $L\left(s, f_{D}\right)$ is given by $(-1)^{d} \chi_{D}\left(\mathfrak{p}^{e}\right)$, which is equal to 1 by the above assumptions.

Let us give a brief account of the paper. In Section 2 among other things, we introduce a Gross curve $X$ and a vector bundle $V$ associated to the quaternion algebra $B$. In Section 3 we construct a Hilbert modular form $g$ of half-integral weight with coefficients in $\operatorname{Pic}(V)$. Let $g_{f}$ be the $f$-isotypical component of $g$, and denote the $D$-th Fourier coefficient of $g_{f}$ by $m_{D}$. Then Section 4 . Theorem 2 gives the main result of this paper, which is that under certain conditions on the odd fundamental discriminants $-D$ the central value of $L\left(s, f_{D}\right)$ is given by

$$
L\left(1 / 2, f_{D}\right)=C_{D} \frac{(f, f)}{\left\langle\nu_{f}, \nu_{f}\right\rangle}\left|m_{D}\right|^{2},
$$

where $C_{D}$ is an explicit positive constant and $\left\langle\nu_{f}, \nu_{f}\right\rangle$ is some height pairing; see Section 4 for the precise statement.

Received by the editors January 12, 2010.

2010 Mathematics Subject Classification. Primary 11F67, 11F37.

(C)2010 American Mathematical Society 
Formula (1.1) can be viewed as a geometric generalization of the well-known result due to Waldspurger [12] (for $F=\mathbb{Q}$ ). A more general but probably less explicit generalization can be found in Shimura [10]. When $F=\mathbb{Q}$, similar results have been obtained by Gross [2] (weight 2) and Hatcher [6] (higher weight). Our approach and results are extensions of theirs.

Now we make a few comments on the assumptions stated above. The oddness of the class number $h(F)$ of $F$ is used to guarantee the passage from the original central value formula, which is stated on $B^{\times} / F^{\times}$in $\left[13\right.$, to the current setting on $B^{\times}$. This assumption may be dropped if one can prove a central value formula directly on $B^{\times}$. The assumption on the level of $f$ is used to eliminate the dependence of the central value formula on the choice of the $\operatorname{Pic}\left(\mathcal{O}_{K_{D}}\right)$ orbit of special points. This assumption can be lifted if either a central value formula involving all special points is obtained, or if the Fourier coefficients of automorphic forms of more general type, such as Jacobi forms or vector-valued forms, are considered (see [4 for a similar situation). The assumption on the parity of $d$ and $e$ is to ensure that $f$ comes from a form on $B^{\times}$under the Jacquet-Langlands correspondence. It might be more natural to start with a form on $B^{\times}$directly.

\section{Gross CURVES AND VECTOR BUNDLES}

Let $B$ be the quaternion algebra over $F$ defined as in Section 1(1) or (2). Let $R$ be a fixed order of discriminant $\mathfrak{p}^{e}$ over $\mathcal{O}_{F}$ in $B$, whose construction can be found for example in [3, Section 3] or [13, Section 3.2]. Notice that $R$ is not unique even up to local conjugation. Let $\widehat{F}=F \otimes_{\mathbb{Z}} \widehat{\mathbb{Z}}, \widehat{B}=B \otimes_{\mathbb{Z}} \widehat{\mathbb{Z}}$ and $\widehat{R}=R \otimes_{\mathbb{Z}} \widehat{\mathbb{Z}}$.

2.1. Gross curve $X$. Let $Y$ be the genus zero curve over $F$ associated to $B$. The points of $Y$ over any $F$-algebra $E$ are given by $Y(E)=\left\{\alpha \in B \otimes_{F} E \mid \alpha \neq 0, \operatorname{Tr}(\alpha)=\right.$ $\mathbf{N}(\alpha)=0\} / E^{\times}$.

The Gross curve $X$ is defined by the double coset

$$
X=B^{\times} \backslash Y \times \widehat{B}^{\times} / \widehat{R}^{\times} .
$$

Let $\widehat{B}^{\times}=\bigcup_{i=1}^{n} B^{\times} g_{i} \widehat{R}^{\times}$be the double coset decomposition. Then there is an isomorphism

$$
X \cong \coprod_{i=1}^{n} \Gamma_{i} \backslash Y=\coprod_{i=1}^{n} X_{i},
$$

where each $\Gamma_{i}=\left(B^{\times} \cap g_{i} \widehat{R}^{\times} g_{i}^{-1}\right) / \mathcal{O}_{F}^{\times}$is a finite group with order denoted by $w_{i}$.

Suppose $D \gg 0$ is such that $K_{D}=F(\sqrt{-D})$ can be embedded into $B$. Then, there is a canonical identification $Y\left(K_{D}\right) \cong \operatorname{Hom}_{F}\left(K_{D}, B\right)$ as follows: for any $f \in \operatorname{Hom}_{F}\left(K_{D}, B\right)$, let $y$ be the image of the unique $K_{D}$-line on the quadric $\{\alpha \in$ $\left.B \otimes_{F} K_{D} \mid \alpha \neq 0, \operatorname{Tr}(\alpha)=\mathbf{N}(\alpha)=0\right\}$ on which conjugation by $f\left(K_{D}^{\times}\right)$acts by multiplication by the character $k \mapsto k / \bar{k}$. Notice that $f\left(K_{D}^{\times}\right)$has two fixed points on $Y\left(K_{D}\right)$, one of which is $y$ and the other one is $\bar{y}$, the image of $y$ under the complex conjugate of $\operatorname{Gal}\left(K_{D} / F\right)$. Special points on $X$ over $K_{D}$ are the images of $Y\left(K_{D}\right) \times \widehat{B}^{\times} / \widehat{R}^{\times}$in $X\left(K_{D}\right)$.

Now suppose further that $-D \in F$ is a fundamental discriminant (Definition 3.2) and $\mathfrak{p} \nmid D$. A special point $x=(y, g) \in X\left(K_{D}\right)$ is said to have discriminant $-D$ if $f\left(K_{D}\right) \cap g \widehat{R} g^{-1}=f\left(\mathcal{O}_{K_{D}}\right)$, where $f: K \rightarrow B$ is the embedding corresponding to $y$. If the component $g$ of $x$ is congruent to $g_{i}$ in $B^{\times} \backslash \widehat{R}^{\times} / \widehat{R}^{\times}$, then the special 
point $x$ lies on the component $X_{i}$. Let $h_{i}(D)$ be the number of embeddings of $\mathcal{O}_{K_{D}}$ into $R_{i}$, modulo conjugation by $R_{i}^{\times}$. Then there are exactly $h_{i}(D)$ special points of discriminant $-D$ on the component $X_{i}$. There is a free action of the group $\operatorname{Pic}\left(\mathcal{O}_{K_{D}}\right) \cong K_{D}^{\times} \backslash \widehat{K}_{D}^{\times} / \widehat{\mathcal{O}}_{K_{D}}^{\times}$on the set of special points of discriminant $-D$ as follows. Let $x=(y, g)$ be a special point of discriminant $-D$ and let $a \in \widehat{K}_{D}^{\times}$. Let $\widehat{f}: \widehat{K}_{D}^{\times} \rightarrow \widehat{B}^{\times}$be the homomorphism induced by the embedding $f: K_{D} \rightarrow B$ that corresponds to $y$. The action of $a$ on $x$ is then given by

$$
x_{a}=(y, \widehat{f}(a) g) .
$$

This action is well-defined and defines a free action of $\operatorname{Pic}\left(\mathcal{O}_{K_{D}}\right)$ on the set of special points of discriminant $-D$; see [2, p. 133]. The argument of [2, p. 133] shows that the orbit set classifies embeddings of $\mathcal{O}_{K_{D}, \mathfrak{p}}$ into $R_{\mathfrak{p}}$ modulo conjugation by $R_{\mathfrak{p}}^{\times}$. The latter set is known [7, Theorem 5.12] or [1] to have cardinality 2. On the other hand, the complex conjugate of $\operatorname{Gal}\left(K_{D} / F\right)$ on $Y\left(K_{D}\right)$ induces an action on special points of discriminant $-D$. Thus the product group $\operatorname{Gal}\left(K_{D} / F\right) \times \operatorname{Pic}\left(\mathcal{O}_{K_{D}}\right)$ acts simply transitively on the set of all special points of discriminant $-D$ on $X$ by counting the number of points.

The group $\operatorname{Pic}(X)$ of line bundles (or linearly equivalent divisor classes) on $X$ is isomorphic to $\mathbb{Z}^{n}$, and is generated by the classes $e_{i}$ of degree 1 on each component $X_{i}$. We define two divisor classes associated to a fundamental discriminant $-D$ (and $\mathfrak{p} \nmid D$ ). Let $u(D)=\left|\mathcal{O}_{K_{D}}^{\times} / \mathcal{O}_{F}^{\times}\right|$. The first one is given by

$$
c_{D}=\frac{1}{2 u(D)} \sum_{\operatorname{disc}(x)=-D}(x),
$$

where the sum is over all special points of discriminant $-D$. The second one is given by the $\operatorname{Pic}\left(\mathcal{O}_{K_{D}}\right)$ orbit

$$
y=\frac{1}{u(D)} \sum_{a \in \operatorname{Pic}\left(\mathcal{O}_{K_{D}}\right)}\left(x_{a}\right) \in \operatorname{Pic}(X),
$$

where $x \in X$ is any fixed special point of discriminant $-D$. Since $\bar{x}$ and $x$ lie on the same component of $X$, we obtain the following equality as divisor classes:

$$
c_{D}=y=\frac{1}{2 u(D)} \sum_{i=1}^{n} h_{i}(D) e_{i} \in \operatorname{Pic}(X)
$$

If $a=\sum_{i=1}^{n} a_{i} e_{i}$ and $b=\sum_{i=1}^{n} b_{i} e_{i}$ are two divisor classes in $\operatorname{Pic}(X)$, the height pairing between them is given by

$$
\langle a, b\rangle=\sum_{i=1}^{n} w_{i} a_{i} b_{i} .
$$

2.2. Vector bundle $V$ over $X$. In the following, let $v=1, \cdots, d$ be the subscripts corresponding to infinite places of $F$. Each local component $B_{v}=B \otimes_{F} F_{v}$ with the standard Hamiltonian quaternion algebra over $\mathbb{R}$.

Let $W=\mathbb{C} x \oplus \mathbb{C} y$ be the standard two-dimensional representation of $\mathrm{SU}(2)$ with an inner product given by $[x, x]=[y, y]=1$ and $[x, y]=0$. For each infinite place $v=1, \cdots, d$, the space $\operatorname{Sym}^{2 k_{v}-2}(W)$ is also an inner product space with basis $\left\{x^{2 k_{v}-2}, x^{2 k_{v}-1} y, \cdots, y^{2 k_{v}-2}\right\}$. The induced inner product on $\operatorname{Sym}^{2 k_{v}-2}(W)$ is such that $\left[x^{i} y^{j}, x^{i} y^{j}\right]=i ! j !$; see [5, Section 3] for more details. Notice that $\operatorname{Sym}^{2 k_{v}-2}(W)$ 
is also a representation of $\mathrm{SO}(3)$, thus can be regarded as a representation of $B_{v}^{\times}$. Let $U_{v}$ be the vector space of trace-free elements of $B_{v}$. Then $B_{v}^{\times}$has a natural representation on $U_{v}$ by conjugation. An inner product on $U_{v}$ is given by $\left[u_{1}, u_{2}\right]=$ $\frac{1}{2} \operatorname{Tr}\left(u_{1} \bar{u}_{2}\right)$. The space $\operatorname{Sym}^{k_{v}-1}\left(U_{v}\right)$, as an inner product space, has an orthogonal decomposition [5, Section 3]

$$
\operatorname{Sym}^{k_{v}-1}\left(U_{v}\right)=\operatorname{Sym}^{2 k_{v}-2}(W) \oplus M_{v} .
$$

Let $W_{2 \mathbf{k}-1}=\prod_{v} \operatorname{Sym}^{2 k_{v}-2}(W)$. The space $W_{2 \mathbf{k}-1}$ has an action of $B^{\times}$through the diagonal embedding $B^{\times} \rightarrow\left(B \otimes_{\mathbb{Q}} \mathbb{R}\right)^{\times}=\prod_{v} B_{v}^{\times}$. The vector bundle $V$ is then defined by

$$
V=B^{\times} \backslash Y \times W_{2 \mathbf{k}-1} \times \widehat{B}^{\times} / \widehat{R}^{\times}
$$

If we write out the double coset quotient, then

$$
V \cong \coprod_{i=1}^{n} \Gamma_{i} \backslash\left(Y \times W_{2 \mathbf{k}-1}\right)=\coprod_{i=1}^{n} V_{i}
$$

For each $i$, let $W_{2 \mathbf{k}-1}^{\Gamma_{i}}$ be the elements invariant under the action of $\Gamma_{i}$. Then $\operatorname{Pic}(V)$ is defined by

$$
\operatorname{Pic}(V)=\bigoplus_{i=1}^{n} \mathbb{C} e_{i} \otimes W_{2 \mathbf{k}-1}^{\Gamma_{i}}
$$

Notice that $\operatorname{Pic}(V)$ is already a vector space over $\mathbb{C}$. Letting $\nu=\left(y, g_{i}, w\right) \in V$, define the class of $\nu$ to be

$$
(\nu)=\frac{1}{\left|\Gamma_{i}\right|} \sum_{\gamma \in \Gamma_{i}} e_{i} \otimes \gamma(w) \in \operatorname{Pic}(V) .
$$

The height pairing on $V \times V$ is defined as follows. Let $\nu_{1}=\left(y_{1}, g_{i}, w_{1}\right)$ and $\nu_{2}=\left(y_{2}, g_{j}, w_{2}\right)$. Then

$$
\left\langle\nu_{1}, \nu_{2}\right\rangle= \begin{cases}0, & i \neq j \\ \sum_{\gamma \in \Gamma_{i}}\left[w_{1}, \gamma\left(w_{2}\right)\right]_{W_{2 \mathbf{k}-1}} . & i=j\end{cases}
$$

Formula (2.13) induces a height pairing on $\operatorname{Pic}(V) \times \operatorname{Pic}(V)$, which coincides with (2.7) if $k_{v}=2$ for all $v$.

Let $D \gg 0$ be such that there is an embedding $f: K_{D} \rightarrow B$. At each infinite place $v$, let $u_{v}^{D}=\sqrt{-D_{v}} \in U_{v}$, and let $w_{v}^{D}$ be the component of $\left(u_{v}^{D}\right)^{k_{v}-1}$ in $\operatorname{Sym}^{2 k_{v}-2}(W)$ through (2.8). Write $w_{0}^{D}=\prod_{v} w_{v}^{D} \in W_{2 \mathbf{k}-1}$. A straight computation [5, p. 556] gives

$$
\left[w_{0}^{D}, w_{0}^{D}\right]=\prod_{v} \frac{2^{2 k_{v}-2} D_{v}^{k_{v}-1}\left(k_{v}-1\right) !^{3}}{\left(2 k_{v}-2\right) !} .
$$

Suppose further that $-D$ is a fundamental discriminant with $\mathfrak{p} \nmid D$. A special point on $V$ of discriminant $-D$ is a point of the form $\nu=\left(y, g, w_{0}^{D}\right)$, where $x=(y, g)$ is a special point on $X$ of discriminant $-D$. The action of $\operatorname{Pic}\left(\mathcal{O}_{K_{D}}\right)$ on special points of $X$ extends naturally to special points of $V$ through $\nu_{a}=\left(x_{a}, w_{0}^{D}\right)$; see (2.3). Similar to (2.6) define the special cycle of $V$ by

$$
\nu_{D}=\frac{1}{u(D)} \sum_{a \in \operatorname{Pic}\left(\mathcal{O}_{K_{D}}\right)}\left(\nu_{a}\right)=\frac{1}{2 u(D)} \sum_{i=1}^{n} h_{i}(D)\left(e_{i} \otimes w_{0}^{D}\right) \in \operatorname{Pic}(V) .
$$


2.3. Curve $X^{\prime}$ and vector bundle $V^{\prime}$. To utilize the central value formula of 13 , we need to define a new curve $X^{\prime}$ and a vector bundle $V^{\prime}$ on it. The construction is parallel to that in Section 2.2. After the construction the relationship between them is studied.

The curve $X^{\prime}$ is defined by

$$
X^{\prime}=B^{\times} \backslash Y \times \widehat{B}^{\times} / \widehat{F}^{\times} \widehat{R}^{\times} .
$$

If we write $\widehat{B}^{\times}=\bigcup_{j=1}^{m} B^{\times} g_{j}^{\prime} \widehat{F}^{\times} \widehat{R}^{\times}$for the double coset decomposition, then

$$
X^{\prime} \cong \coprod_{j=1}^{m} \Gamma_{j}^{\prime} \backslash Y,
$$

where $\Gamma_{j}^{\prime} \cong\left(B^{\times} \cap g_{j}^{\prime} \widehat{F}^{\times} \widehat{R}^{\times} g_{j}^{\prime-1}\right) / F^{\times}$.

Thus the group of line bundles $\operatorname{Pic}\left(X^{\prime}\right)$ of $X^{\prime}$ is isomorphic to $\mathbb{Z}^{m}$. If $a^{\prime}=$ $\sum_{j=1}^{m} a_{j} e_{j}^{\prime}$ and $b^{\prime}=\sum_{j=1}^{m} b_{j} e_{j}^{\prime}$, the height pairing between them is given by

$$
\left\langle a^{\prime}, b^{\prime}\right\rangle^{\prime}=\sum_{j=1}^{m} w_{j}^{\prime} a_{j} b_{j} .
$$

Assumption. From now on we will assume that the class number $h(F)$ of $F$ is odd.

Lemma 2.1. The (multiplication) action of $\operatorname{Pic}\left(\mathcal{O}_{F}\right)=F^{\times} \backslash \widehat{F}^{\times} / \widehat{\mathcal{O}}_{F}^{\times}$on $S=$ $B^{\times} \backslash \widehat{B}^{\times} / \widehat{R}^{\times}$is free. The quotient space is given by

$$
B^{\times} \backslash \widehat{B}^{\times} / \widehat{F}^{\times} \widehat{R}^{\times} \text {. }
$$

Proof. The second claim is obvious, and we only need to prove the first one. Let $t \in \widehat{F}^{\times}$be a stabilizer of $g \in \widehat{B}^{\times}$such that $g t=a g r$ with $a \in B^{\times}$and $r \in \widehat{R}^{\times}$. Then $\mathbf{N}(g) \mathbf{N}(t)=\mathbf{N}(a) \mathbf{N}(g) \mathbf{N}(r)$, which implies that $t^{2}=\mathbf{N}(t)=\mathbf{N}(a) \mathbf{N}(r) \in F^{\times} \widehat{\mathcal{O}}_{F}^{\times}$. Since $h(F)$ is assumed to be odd, we conclude that $t$ itself has to define a trivial class in $\operatorname{Pic}\left(\mathcal{O}_{F}\right)$.

By Lemma 2.1 we can and will take $g_{j}^{\prime}=g_{j}$ for $j=1, \cdots, m$. Also, $n=m h(F)$.

Remark 2.1. The same argument shows that the natural homomorphism $\operatorname{Pic}\left(\mathcal{O}_{F}\right) \rightarrow$ $\operatorname{Pic}\left(\mathcal{O}_{K}\right)$ is injective for any quadratic extension $K / F$.

Lemma 2.2. For each $j=1, \cdots, m$, there is a natural isomorphism from $\Gamma_{j}$ to $\Gamma_{j}^{\prime}$. More precisely, the natural homomorphism

$$
\left(B^{\times} \cap g_{j} \widehat{R}^{\times} g_{j}^{-1}\right) / \mathcal{O}_{F}^{\times} \cong\left(B^{\times} \cap g_{j} \widehat{F}^{\times} \widehat{R}^{\times} g_{j}^{-1}\right) / F^{\times}
$$

is an isomorphism. Thus $w_{j}^{\prime}=w_{j}$ for $j=1, \cdots, m$.

Proof. The homomorphism in (2.19) is induced by the identity map. To see that it is surjective, suppose $b \in B^{\times} \cap g_{j} \widehat{F}^{\times} \widehat{R}^{\times} g_{j}^{-1}$. Then we need to find $b_{1} \in B^{\times} \cap g_{j} \widehat{R}^{\times} g_{j}^{-1}$ such that $b_{1}=b f$ for some $f \in F^{\times}$. Since $h(F)$ is odd, and taking the norms of $b=g_{j} t r g_{j}^{-1} \in B^{\times}$we get $\mathbf{N}(b)=t^{2} \mathbf{N}(r) \in F^{\times}$, which implies that $t \in F^{\times} \widehat{\mathcal{O}}_{F}^{\times}$, so $b_{1}=b f$ for some $f \in F$. For injectivity, suppose $b_{1}, b_{2} \in B^{\times} \cap g_{j} \widehat{R}^{\times} g_{j}^{-1}$ such that $b_{1}=f b_{2}$. Since $b_{1} b_{2}^{-1} \in g_{j} \widehat{R}^{\times} g_{j}^{-1}$, we get that $f \in F^{\times} \cap g_{j} \widehat{R}^{\times} g_{j}^{-1}=\mathcal{O}^{\times}$. 
Corollary 2.3. The natural projection

$$
\pi: X=B^{\times} \backslash Y \times \widehat{B}^{\times} / \widehat{R}^{\times} \rightarrow X^{\prime}=B^{\times} \backslash Y \times \widehat{B}^{\times} / \widehat{F}^{\times} \widehat{R}^{\times}
$$

is an étale covering of equal degree $h(F)$.

Corollary 2.4. Let $c_{D}^{\prime}=\pi\left(c_{D}\right) \in \operatorname{Pic}\left(X^{\prime}\right)$. Then

$$
\pi^{*}\left(c_{D}^{\prime}\right)=c_{D}
$$

Thus $\left\langle c_{D}, c_{D}\right\rangle=\operatorname{deg}(\pi)\left\langle c_{D}^{\prime}, c_{D}^{\prime}\right\rangle^{\prime}=h(F)\left\langle c_{D}^{\prime}, c_{D}^{\prime}\right\rangle^{\prime}$ by the projection formula.

Notice that the centers of $(B \otimes \mathbb{R})^{\times}$and $B_{v}^{\times}$act trivially on $W_{2 \mathbf{k}-1}$ and $U_{v}$ (Section 2.2) respectively. Thus the vector bundle $V$ descends to a vector bundle on $X^{\prime}$ and is denoted by $V^{\prime}$. The group $\operatorname{Pic}\left(V^{\prime}\right)$ can also be defined similarly. $\operatorname{Pic}(V)$ is equipped with a height pairing, denoted again by $\langle\cdot, \cdot\rangle^{\prime}$. The projection of a special point $\nu$ of fundamental discriminant $-D$ on $V$ defines a special point $\nu^{\prime}$ on $V^{\prime}$. Hence the special cycle $\nu_{D}(2.15)$ descends to a special cycle on $V$ :

$$
\nu_{D}^{\prime}=\sum_{a \in \operatorname{Pic}\left(\mathcal{O}_{K_{D}}\right) / \operatorname{Pic}\left(\mathcal{O}_{F}\right)}\left(\nu_{a}^{\prime}\right)=\frac{1}{2 u(D)} \sum_{j=1}^{m} h_{j}(D)\left(e_{j}^{\prime} \otimes w_{0}^{D}\right) \in \operatorname{Pic}\left(V^{\prime}\right) .
$$

Similar to Corollary 2.4, we have the following.

Proposition 2.5. Let $\nu_{D}$ and $\nu_{D}^{\prime}$ be defined in (2.15) and (2.21) respectively. Then

$$
\left\langle\nu_{D}, \nu_{D}\right\rangle=h(F)\left\langle\nu_{D}^{\prime}, \nu_{D}^{\prime}\right\rangle^{\prime} .
$$

\section{Hilbert modular forms of HalF-INTEgRAl Weight}

Let $B^{0}$ be the subspace of trace-free elements of $B$, and let $U=\prod_{v} B^{0} \otimes_{v} \mathbb{R}$. For each infinite place $v$, let $\mu_{v}^{1}, \mu_{v}^{2}, \mu_{v}^{3}$ be a fixed basis of $B^{0} \otimes_{v} \mathbb{R}$. A homogeneous polynomial $P=\prod_{v} P_{v}$ of degree $\mathbf{k}-1$ on $U$ is said to be spherical harmonic if for each $v$,

$$
\sum_{i=1}^{3} \frac{\partial^{2}}{\partial x_{i, v}^{2}} P_{v}=0
$$

Lemma 3.1. For each $\nu \in \operatorname{Pic}(V)$, there is a spherical harmonic polynomial $P_{i}^{\nu}=$ $\prod_{v} P_{i, v}^{\nu}$ of degree $\mathbf{k}-1$ on $U$ such that for every $D \gg 0$, one has

$$
\left\langle\nu,\left(e_{i} \otimes w_{0}^{D}\right)\right\rangle=\prod_{v} P_{i, v}^{\nu}\left(\sqrt{-D_{v}}\right)=\prod_{v} P_{i, v}^{\nu}\left(x_{1, v}, x_{2, v}, x_{3, v}\right),
$$

where $\sqrt{-D_{v}}=x_{1, v} \mu_{v}^{1}+x_{2, v} \mu_{v}^{2}+x_{3, v} \mu_{v}^{3}$.

Proof. Here $w_{0}^{D}$ is defined in Section 2.2, See 9, Proposition 2.10] or [5, Lemma 1] for a proof.

Let $R_{i}, i=1, \cdots, n$ be the order associated to $g_{i} \in B^{\times} \backslash \widehat{B}^{\times} / \widehat{R}^{\times}$, that is, $R_{i}=$ $B \cap g_{i} \widehat{R} g_{i}^{-1}$. Let

$$
S_{i}=\mathcal{O}_{F}+2 R_{i}
$$

be a suborder of $R_{i}$. Write $S_{i}^{0}$ for the subset of trace-free elements of $S_{i}$. For each $D \gg 0$ in $\mathcal{O}_{F}$, define $A_{i}(D)=\left\{b \in S_{i}^{0} \mid \mathbf{N}(b)=-b^{2}=D\right\}$ and $a_{i}(D)=\left|A_{i}(D)\right|$. 
Definition 3.2. Let $D \gg 0$ be in $\mathcal{O}_{F}$ and $K_{D}=F(\sqrt{-D})$. Then $-D$ is called a fundamental discriminant if $\mathcal{O}_{K_{D}}$ has relative discriminant $(D)$ over $\mathcal{O}_{F}$, and

$$
\mathcal{O}_{K}=\mathcal{O}_{F}+\frac{a+\sqrt{-D}}{2} \mathcal{O}_{F}
$$

for some $a \in \mathcal{O}_{F}$.

Proposition 3.3. Suppose the relative discriminant of $K_{D}$ over $F$ is $(D)$, and suppose $K_{D}$ splits over all primes of $F$ dividing 2 . Then $-D$ is a fundamental discriminant.

Proof. First, notice that $x=\frac{a+\sqrt{-D}}{2}$ for $a \in \mathcal{O}_{F}$ is in $\mathcal{O}_{K_{D}}$ if and only if $4 \mid\left(a^{2}+D\right)$.

Let $(2)=\mathfrak{p}_{1}^{e_{1}} \cdots \mathfrak{p}_{g}^{e_{g}}$ be the prime decomposition of (2) in $\mathcal{O}_{F}$. Since each $\mathfrak{p}_{i}$ splits in $K_{D}$, the equation $y_{i}^{2}=-D$ has solutions in $\mathcal{O}_{F, \mathfrak{p}_{i}}$, which modulo $\mathfrak{p}_{i}^{2 e_{i}}$ implies that $y_{i}^{2} \equiv-D\left(\bmod \mathfrak{p}_{i}^{2 e_{i}}\right)$ has solutions for all $i$. Now the Chinese Remainder Theorem tells us that there is an $a \in \mathcal{O}_{F}$ such that $a \equiv y_{i}$ for all $i$. Such an $a$ satisfies the required property $a^{2} \equiv-D(\bmod 4)$. Hence $x=\frac{a+\sqrt{-D}}{2} \in \mathcal{O}_{K_{D}}$.

Let $\mathcal{O}=\mathcal{O}_{F}+\frac{a+\sqrt{-D}}{2} \mathcal{O}_{F}$. As $\left(\frac{a+\sqrt{-D}}{2}\right)^{2}=\frac{a^{2}-D}{4}+\frac{a \sqrt{-D}}{2}=\frac{-a^{2}-D}{4}+a \frac{a+\sqrt{-D}}{2} \in$ $\mathcal{O}$, so $\mathcal{O}$ is an order in $K$. Moreover, the discriminant of $\mathcal{O}$ is $(D)$, which is the same as that of $\mathcal{O}_{K}$. Therefore $\mathcal{O}_{K}=\mathcal{O}=\mathcal{O}_{F}+\frac{a+\sqrt{-D}}{2} \mathcal{O}_{F}$ and $-D$ is a fundamental discriminant.

Proposition 3.3 implies that there are plenty of fundamental discriminants. For instance, let $D$ be square-free such that $\left(D, d_{F}\right)=1$ and $-D \equiv 1(\bmod 8)$. Then $-D$ is a fundamental discriminant.

Proposition 3.4. Suppose $-D$ is a fundamental discriminant. Then, for each $i=1, \cdots, n$,

$$
\frac{a_{i}(D)}{w_{i}}=\frac{h_{i}(D)}{u(D)},
$$

where $h_{i}(D)$ is the number of (optimal) embeddings of $\mathcal{O}_{K}$ into $R_{i}$, modulo conjugation by $R_{i}^{\times}$.

Proof. First let $f: \mathcal{O}_{K} \rightarrow R_{i}$ be an embedding. Write $\mathcal{O}_{K}=\mathcal{O}_{F}+\frac{a+\sqrt{-D}}{2} \mathcal{O}_{F}$. The element $b=f(\sqrt{-D}) \in R_{i}$ then satisfies $\operatorname{Tr}(b)=0$ and $\mathbf{N}(b)=-D$. Since $\frac{a+b}{2}=f\left(\frac{a+\sqrt{-D}}{2}\right) \in R_{i}$, we have $b \in A_{i}(D)$.

Conversely let $b \in A_{i}(D)$. Thus $a^{\prime}+b \in 2 R_{i}$ for some $a^{\prime} \in \mathcal{O}_{F}$. In particular, $\frac{a^{\prime}+b}{2}$ is integral over $\mathcal{O}_{F}$, which implies that $\frac{a^{\prime}+\sqrt{-D}}{2} \in K_{D}$ is integral over $K_{D}$, that is, $\frac{a^{\prime}+\sqrt{-D}}{2} \in \mathcal{O}_{K}$. By comparing discriminants we get

$$
\mathcal{O}_{K}=\mathcal{O}_{F}+\frac{a^{\prime}+\sqrt{-D}}{2} \mathcal{O}_{F}
$$

Thus we obtain an embedding $f: \mathcal{O}_{K} \rightarrow R_{i}$ by letting $f\left(\frac{a^{\prime}+\sqrt{-D}}{2}\right)=\frac{a^{\prime}+b}{2}$.

The group $\Gamma_{i}=R_{i}^{\times} / \mathcal{O}_{F}^{\times}$acts on $A_{i}(D)$ and the set of embeddings by conjugation. Hence we have proved that

$$
\left|A_{i}(D) / \Gamma_{i}\right|=h_{i}(D) .
$$

Now (3.2) follows form (3.3) because the order of the stabilizer of an element $b \in$ $A_{i}(D)$ under the action of $\Gamma_{i}$ is equal to $u(D)$. 
Proposition 3.5. The series

$$
g=\sum_{D \gg 0, \text { or } D=0}\left(\sum_{i=1}^{n} \frac{a_{i}(D)}{2 w_{i}}\left(e_{i} \otimes w_{0}^{D}\right)\right) q^{D}
$$

defines a Hilbert modular form of weight $\mathbf{k}+\frac{1}{2}$ with coefficients in $\operatorname{Pic}(V)$. Here $z=\left(z_{1}, z_{2}, \cdots, z_{d}\right) \in \mathbb{H}^{d}$, and $q^{D}=\exp (2 \pi i \operatorname{Tr} D z)$. Moreover, if $-D$ with $\mathfrak{p} \nmid D$ is a fundamental discriminant, then the D-th Fourier coefficient of $g$ equals $\nu_{D}$.

Proof. By Lemma 3.1, the $i$-th series in (3.4) is a theta series with spherical harmonic coefficients attached to the quadratic space $S_{i}^{0}$. The modularity and weight of such a series is well known when $F=\mathbb{Q}$; see for instance 8]. For a general field $F$, the proof can be found in [11, Section 5].

If $-D$ is a fundamental discriminant, the equality between the $D$-th Fourier coefficient of $g$ and $\nu_{D}$ follows by comparing (2.15) and (3.2).

The term for $D=0$ in (3.4) is nonzero only if $k_{v}=2$ for all $v$.

\section{Central values and Fourier coefficients \\ OF HALF-INTEGRAL WEIGHT FORMS}

In this section we first recall a formula for the central value $L\left(1 / 2, f_{D}\right)$ which is expressed in terms of the height pairing between special cycles of $\operatorname{Pic}(V)$. Then we relate these central values to the Fourier coefficients of the Hilbert modular form of half-integral weight constructed in Proposition 3.5

From now on let $-D$ with $\mathfrak{p} \nmid D$ be a fundamental discriminant, and assume there exists a special point of discriminant $-D$ on $X$.

Theorem 1. The central value of $L\left(s, f_{D}\right)$ is given by

$$
L\left(1 / 2, f_{D}\right)=C_{D}(f, f)\left\langle\nu_{D, f}, \nu_{D, f}\right\rangle,
$$

where $C_{D}$ is given by

$$
C_{D}=C_{1} h(F) d_{F}^{3 / 2} d_{K_{D}}^{-1 / 2} \prod_{v} \frac{\left(k_{v}-1\right) !}{2 D_{v}^{1-k_{v}}}
$$

with $C_{1}$ defined in [13, (1.4)], $(f, f)$ is the Petersson inner product, and $\nu_{D, f}$ is the $f$-isotypical component of $\nu_{D}$.

Proof. By [13, Theorem 1.2],

$$
L\left(1 / 2, f_{D}\right)=C_{1} d_{F}^{3 / 2} d_{K_{D}}^{-1 / 2}(f, f)\left\langle\nu_{D, f}^{\prime}, \nu_{D, f}^{\prime}\right\rangle_{G},
$$

where $C_{1}$ is the rational number given by [13, (1.4)] and $\langle\cdot, \cdot\rangle_{G}$ is the geometric pairing defined in [13, Section 3.1] through a multiplicity function $M_{\infty}=\prod_{v} M_{v}$, which is defined as follows. Choose a decomposition $B=K_{D}+K_{D}$, and let $\xi(a+b \jmath)=\frac{\mathbf{N}(b \jmath)}{\mathbf{N}(a+b \jmath)}$. Then

$$
M_{v}\left(\gamma_{v}\right)=\frac{2^{2 k_{v}-1}\left(k_{v}-1\right) !^{2}}{\left(2 k_{v}-2\right) !} P_{k_{v}-1}\left(1-2 \xi\left(\gamma_{v}\right)\right),
$$

where $P_{n}(t)=\sum_{j=0}^{n}\left(\begin{array}{c}n \\ j\end{array}\right)\left(\begin{array}{c}n+j \\ n\end{array}\right)\left(\frac{t-1}{2}\right)^{j}$ is the standard $n$-th Legendre polynomial. 
By [5. (7.6)] one has

$$
\begin{aligned}
{\left[w_{0}^{D}, \gamma\left(w_{0}^{D}\right)\right] } & =\left(\prod_{v} P_{k_{v}-1}\left(1-2 \xi\left(\gamma_{v}\right)\right)\right)\left[w_{0}^{D}, w_{0}^{D}\right] \\
& =\prod_{v} P_{k_{v}-1}\left(1-2 \xi\left(\gamma_{v}\right)\right) \cdot \prod_{v} \frac{2^{2 k_{v}-2} D_{v}^{k_{v}-1}\left(k_{v}-1\right) !^{3}}{\left(2 k_{v}-2\right) !} \\
& =\left(\prod_{v} \frac{\left(k_{v}-1\right) !}{2 D_{v}^{1-k_{v}}}\right) M_{\infty}(\gamma) .
\end{aligned}
$$

Comparing the height pairing (2.13) and the geometric pairing in [13, Section 3.1], we obtain the following relation between them:

$$
\left\langle\nu_{D, f}^{\prime}, \nu_{D, f}^{\prime}\right\rangle^{\prime}=\left(\prod_{v} \frac{\left(k_{v}-1\right) !}{2 D_{v}^{1-k_{v}}}\right)\left\langle\nu_{D, f}^{\prime}, \nu_{D, f}^{\prime}\right\rangle_{G} .
$$

By Proposition 2.5.

$$
\left\langle\nu_{D, f}, \nu_{D, f}\right\rangle=h(F)\left(\prod_{v} \frac{\left(k_{v}-1\right) !}{2 D_{v}^{1-k_{v}}}\right)\left\langle\nu_{D, f}^{\prime}, \nu_{D, f}^{\prime}\right\rangle_{G}
$$

which combined with (4.3) completes the proof of (4.1).

Remark 4.1. (1) Strictly speaking, the special cycle in 13 is defined over a curve associated to the group $G=B^{\times} / F^{\times}$, but there is an isomorphism

$$
B^{\times} \backslash \widehat{B}^{\times} / \widehat{F}^{\times} \widehat{R}^{\times} \cong G(F) \backslash G\left(\mathbb{A}_{f}\right) / \Delta,
$$

with $\Delta=\widehat{R}^{\times} / \widehat{\mathcal{O}}_{F}^{\times}$. This isomorphism is induced by $\widehat{B}^{\times} / \widehat{F}^{\times} \cong G\left(\mathbb{A}_{f}\right)$ and $\widehat{R}^{\times} \cap$ $\widehat{F}^{\times}=\widehat{\mathcal{O}}_{F}^{\times}$.

(2) The existence of a special point of discriminant $-D$ implies that the functional equation of $L\left(s, f_{D}\right)$ has sign +1 (due to the assumption on the parity of $d$ and $e$ ). Hence the result of [13 can be applied.

(3) There are naturally defined Hecke operators acting on all the curves and line bundles defined in Section 2. By the Jacquet-Langlands correspondence, $f$ determines an eigenform on the group $B^{\times} \backslash B^{\times}\left(\mathbb{A}_{F}\right)$, which has the same Hecke eigenvalues as $f$. Hence it is legitimate to speak of $f$-isotypical components on $\operatorname{Pic}(V)$.

Let $\nu_{f} \in \operatorname{Pic}(V) \otimes \mathbb{C}$ be a nonzero element in the $f$-isotypical component. By the strong multiplicity-one theorem, such a $\nu_{f}$ is unique up to a scalar multiple. Let

$$
g\left(\nu_{f}\right)=\sum_{D} m_{D} q^{D}=\left\langle g, \nu_{f}\right\rangle .
$$

Now we state the main result of this paper.

Theorem 2. Let $-D$ with $\mathfrak{p} \nmid D$ be an odd fundamental discriminant such that there is a special point on $X$ of discriminant $-D$. Then

$$
L\left(1 / 2, f_{D}\right)=C_{D} \frac{(f, f)}{\left\langle\nu_{f}, \nu_{f}\right\rangle}\left|m_{D}\right|^{2},
$$

where $C_{D}$ is given by (4.2). 
Proof. By Proposition 3.5 and 4.8),

$$
m_{D}=\left\langle\nu_{D}, \nu_{f}\right\rangle=\left\langle\nu_{D, f}, \nu_{f}\right\rangle
$$

Thus

$$
\nu_{D, f}=\frac{m_{D}}{\left\langle\nu_{f}, \nu_{f}\right\rangle} \nu_{f} \in \operatorname{Pic}(V)
$$

So

$$
\left\langle\nu_{D, f}, \nu_{D, f}\right\rangle=\frac{\left|m_{D}\right|^{2}}{\left\langle\nu_{f}, \nu_{f}\right\rangle} .
$$

Now (4.9) follows immediately from (4.1).

The form $g\left(\nu_{f}\right)$ is a Niwa-Shintani lifting of $f$. Hence (4.9) can be regarded as an explicit geometric generalization of the result of [12].

\section{REFERENCES}

1. J. Brzezinski, On embedding numbers into quaternion orders, Comment. Math. Helv. 66 (1991), no. 2, 302-318. MR1107843 (92e:11133)

2. B. Gross, Heights and the special values of L-series, Number theory (Montreal, Que., 1985), CMS Conf. Proc., vol. 7, Amer. Math. Soc., Providence, RI, 1987, pp. 115-187. MR894322 (89c:11082)

3. , Local orders, root numbers, and modular curves, Amer. J. Math. 110 (1988), no. 6, 1153-1182. MR970123 (90b:11053)

4. B. Gross, W. Kohnen, and D. Zagier, Heegner points and derivatives of L-series. II, Math. Ann. 278 (1987), no. 1-4, 497-562. MR909238(89i:11069)

5. R. Hatcher, Heights and L-series, Canad. J. Math. 42 (1990), no. 3, 533-560. MR1062744 (92b:11031)

6. (1994), no. 3, 683-688. MR1233973 (95a:11045)

7. H. Hijikata, A. Pizer, and T. Shemanske, Orders in quaternion algebras, J. Reine Angew. Math. 394 (1989), 59-106. MR977435(90d:11128)

8. W. Pfetzer, Die Wirkung der Modulsubstitutionen auf mehrafache Thetareihen zu quadratischen Formen ungerader Variablenzahl, Arch. Math. (Basel) 4 (1953), 448-454. MR0059945 (15:603d)

9. A. Pizer, An algorithm for computing modular forms on $\Gamma_{0}(N)$, J. Algebra 64 (1980), no. 2, 340-390. MR579066 (83g:10020)

10. G. Shimura, On the Fourier coefficients of Hilbert modular forms of half-integral weight, Duke Math. J. 71 (1993), no. 2, 501-557. MR.1233447 (94e:11046)

11. _ On the transformation formulas of theta series, Amer. J. Math. 115 (1993), no. 5, 1011-1052. MR 1246183 (94h:11045)

12. J.-L. Waldspurger, Sur les coefficients de Fourier des formes modulaires de poids demi-entier, J. Math. Pures Appl. (9) 60 (1981), no. 4, 375-484. MR646366 (83h:10061)

13. H. Xue, Central values of Rankin L-functions, Int. Math. Res. Not. (2006), Art. ID 26150, 41. MR2249999 (2008e:11059)

Department of Mathematical Sciences, Clemson University, Clemson, South CaroLINA 29634

E-mail address: huixue@clemson.edu 